\title{
Hábitos lectores y titulación de los padres en la percepción de la competencia comunicativa de futuros maestros
}

The influence of parents' reading habits and educational level on the perception of communicative competence in preservice teachers

\author{
Beatriz Sierra-Arizmendiarrieta ${ }^{1}$ \\ bsierra@uniovi.es \\ María Del Rosario Neira-Piñeiro \\ neiramaria@uniovi.es \\ Universidad de Oviedo, España
}

\section{Resumen:}

INTRODUCCIÓN. El objetivo principal de este trabajo es determinar en qué medida las variables "afición a la lectura en la familia" y "titulación de los padres" pueden incidir en la percepción de la competencia comunicativa de los estudiantes universitarios participantes en el estudio. MÉTODO. Se parte de una muestra de 361 estudiantes del Grado de Maestro de Educación Primaria de las universidades de Jaén y Oviedo y se realiza un estudio descriptivo-transversal consistente en un análisis de contingencia. Se distinguen tres unidades de competencia, obtenidas por el análisis factorial realizado: comunicación oral efectiva en el aula, adecuación del registro y producción eficaz de textos escritos. RESULTADOS. Los resultados evidencian una clara incidencia de la variable "afición a la lectura en la fami-

\begin{abstract}
:
INTRODUCTION. This study aims to know to what extent the variables 'love of reading in the family' and 'parents' educational level' may have an impact on the perception of communicative competence in university students participating in this study. METHOD. 361 Primary Education Degree students coming from two Spanish universities participated in the study. A descriptive-transversal design was used for the study consisting of a contingency analysis. Three competence units were defined through factorial analysis: effective oral communication in the classroom, register adequacy and effective production of written texts. RESULTS. The results clearly show the influence of the variable 'love of reading in the family' in the analyzed dimensions. On the contrary, no relation is detected between these di-
\end{abstract}

1 Dirección para correspondencia (correspondence address):

Beatriz Sierra-Arizmendiarrieta. C/Marqués de Santa Cruz, 7-7ºD. 33007- Oviedo (España). 
Hábitos lectores y titulación de los padres en la percepción de la competencia comunicativa de futuros maestros

Beatriz Sierra-Arizmendiarrieta y María Del Rosario Neira-Piñeiro

lia" en las dimensiones de competencia analizadas, no dándose ninguna relación entre éstas y la variable "titulación de los padres". DISCUSIÓN. El hecho de que esta variable no tenga la misma incidencia puede deberse precisamente a que no es una variable que sirva de modelo en el ejercicio, al no estar directamente relacionada con la práctica de la competencia analizada.

\section{Palabras clave:}

Competencia comunicativa; contexto familiar; hábito lector; formación de profesores. mensions and the variable 'parents' educational level'. DISCUSSION. The fact that this variable does not have the same incidence can be explained, precisely, because it is does not function as a model, as it is not directly related to the practice of the competence analyzed.

\section{Key words:}

Communicative competence; family environment; reading habits; teacher education.

\section{Résumé:}

INTRODUCTION. Le principal objectif de ce travail est de déterminer dans quelle mesure les variables "goût de la lecture dans la famille" et "niveau d'études des parents" peuvent avoir une incidence sur la perception de la compétence communicative des étudiants universitaires qui ont participé à cette recherche. MÉTHODE. Partant d'un échantillon de 361 étudiants de la Licence en Enseignement Primaire des Universités de Jaen et Oviedo, une étude descriptive-transversale consistant en une analyse de contingence a été réalisée. Trois unités de compétence, obtenues par une analyse factorielle: communication orale effective en classe, adéquation du registre et production efficace de textes écrits ont été établies. RÉSULTATS. Les résultats mettent en évidence une claire incidence de la variable "goût de la lecture dans la famille" dans les dimensions de compétence analysées, tandis qu'il n'y a pas de relation entre celles-ci et la variable "niveau d'études des parents". DISCUSSION. Le fait que cette variable n'ait pas la même incidence peut s'expliquer, justement, parce qu'elle ne sert pas de modèle, n'étant pas directement liée à la pratique de la compétence analysée.

\section{Mots clés:}

Compétence communicative; contexte familial; habitudes de lecture; formation des maîtres.

Fecha de recepción: 20-05-2020

Fecha de aceptación: 03-03-2020

\section{Introducción}

El contexto familiar es uno de los factores que inciden en el rendimiento académico y escolar de los estudiantes (Alarcón Montiel, Piña Osorio, García Valcárcel y Tejedor, 2019; Castro, Expósito-Casas, López-Martín, Lizasoain, Navarro-Asencio y Gaviria, 2015; Córdoba, García, Luengo, Vizuete y Feu, 2011), extendiéndose su influjo al progreso lingüístico de niños y jóvenes a lo largo de su formación académico-escolar. Así, el desarrollo lingüístico en las primeras edades se correlaciona con el nivel 
socio-económico familiar, resultando determinantes las primeras experiencias con el lenguaje en el hogar (Hart y Risley, 1995, citados en Margallo y Mata, 2015). De igual modo, un entorno familiar alfabetizado, con padres que sean modelos lectores y donde se realicen prácticas de lectura compartida, incide positivamente en el desarrollo del hábito lector de los menores y en su competencia lecto-escritora, así como en su progreso lingüístico en general (García-Fontes, 2012; Gil, 2009; Serna, Rodríguez, y Etxaniz, 2017; Yubero y Larrañaga, 2010). De modo más general, el nivel de estudios de los padres es una de las variables que se suelen tener en cuenta en las investigaciones sobre la influencia del contexto familiar en el rendimiento académico de los menores (Fajardo, Maestre, Castaño, León y Polo, 2017; Bravo, Salvo, Mieres, Mansilla y Hederich, 2017).

Si bien cada día cobra mayor interés el desarrollo de las competencias genéricas y específicas en el alumnado universitario, resulta especialmente relevante -por su transversalidad y su importancia en cualquier contexto laboral-, la competencia comunicativa. Una reciente investigación con una amplia muestra de estudiantes, egresados y profesores de 24 universidades españolas, resalta la necesidad de valorar en la Formación Inicial del Profesorado la presencia de competencias relacionadas con la "comunicación oral y escrita" (Romero, Castejón-Oliva, López y Fraile, 2017). Otros estudios de carácter cualitativo destacan la competencia comunicativa como "determinante y nuclear del profesional de la educación" (Domínguez, Medina y López, 2018, p. 57).

La revisión de la bibliografía lleva, por tanto, a plantear como problema de investigación "en qué medida los hábitos lectores de los padres y su nivel académico inciden en la percepción de la competencia comunicativa de estudiantes de Maestro en Educación Primaria". De este modo, se ha definido las variables "afición a la lectura en la familia" y "titulación de los padres", dado que ambas pueden incidir en la competencia comunicativa de los sujetos y, en el primer caso, en las destrezas de lectura y escritura. Este problema condicionará, como se verá, los objetivos de la investigación.

\section{Marco teórico}

La competencia comunicativa, entendida como la capacidad de comunicarse eficaz y adecuadamente en diferentes contextos (Hymes, 1971; 
Gumperz y Hymes, 1972), es esencial para cualquier individuo, formando parte de las competencias profesionales que se pretenden desarrollar en la Educación Superior (Clemente-Ricolfe y Escribá-Pérez, 2013; Luengo, Perea y García, 2013). Además, resulta indispensable para el adecuado ejercicio de la docencia (Castellà, Comelles, Cros y Vilà, 2007), siendo por tanto imprescindible para los futuros maestros (Aguilar, 2013). Por ello, numerosas investigaciones han analizado el nivel de competencia comunicativa del alumnado universitario en general (Conchado y Carot, 2013; España, 2011; Guzmán-Simón y García-Jiménez, 2014), y de los estudiantes de Pedagogía y Magisterio en particular (Domingo, Gallego, García y Rodríguez, 2010; Gallego y Rodríguez, 2014 y 2015; Mayoral, Timoneda y Pérez, 2013; Rico y Níkleva, 2016; Rodríguez, Ayllón, Gallego y Gómez 2017; Rodríguez, 2011).

Entre las diferentes dimensiones que integran la competencia comunicativa en los futuros docentes, esta investigación ${ }^{2}$ se centra en tres unidades de competencia definidas a partir del análisis factorial realizado:

- Factor 1: Comunicación oral efectiva en el aula

- Factor 2: Adecuación del registro

- Factor 3: Producción eficaz de textos escritos

\section{Factor 1: Comunicación oral efectiva en el aula}

Este primer factor se centra en las habilidades de comunicación oral, un aspecto esencial dentro de la competencia comunicativa (Níkleva y López-García, 2019). Agrupa siete indicadores: la vocalización y regulación de la velocidad en cualquier intervención oral en el aula, la utilización de un volumen adecuado y audible para todos los destinatarios, el uso de un tono que muestre seguridad, la modulación de la voz para remarcar diferentes partes del discurso y motivar a los oyentes, la mirada como recurso interactivo, la capacidad de poner distintos ejemplos para el mismo contenido en función de los interlocutores, y la capacidad de persuasión a través del discurso. Podemos agrupar los siete indicadores en tres dimensiones relativas, respectivamente, a los aspectos paralin-

2 Este trabajo se enmarca en un Proyecto de Investigación de tres años (2016-2019), financiado por el MEC, con el título EDECOMEP, "Evaluación y desarrollo de dos competencias genéricas en estudiantes del primer año del Grado de Maestro en Educación Primaria", y con referencia EDU2915-70491-R. 
güísticos (los cuatro primeros indicadores), cinésicos o de lenguaje corporal (el quinto), y discursivos (los dos últimos).

La velocidad, el volumen, el tono y, en general, el control de la voz son elementos que influyen mucho en la percepción del mensaje de los oyentes. Los cuatro indicadores incluidos en esta dimensión están relacionados intrínsecamente en un buen discurso. Respecto a los dos primeros, Sanz (2005) advierte que la velocidad ha de adaptarse al ritmo de comprensión de los interlocutores, que varía en función de la edad o la complejidad del discurso. Además, son importantes las pausas y los silencios para remarcar ideas o señalar puntos clave. Especialmente en la docencia son necesarias una pronunciación y vocalización que eviten errores y faciliten la comprensión. El volumen tendrá que variar de acuerdo al tamaño del aula y el número de personas que en ella se encuentren. El problema más común respecto a un mal empleo del tono es la monotonía que puede producirse por apatía entonativa y por repetición entonativa (Vilà y Castellà, 2014).

Por su parte, la Cinésica es importante porque los enunciados pueden cambiar de sentido comunicativo en función del lenguaje corporal que les acompañe. En nuestra investigación nos hemos fijado únicamente, debido a su importancia para la docencia, en la capacidad de mantener el contacto visual, pues ello contribuye al éxito de la comunicación (Camacho y Sáenz, 2000). Varios trabajos (Miller, 2005; McCroskey y Richmond, 2006) destacan la potencialidad de los recursos no-verbales para generar actitudes positivas hacia la escuela. Cuadrado (2008) concluye en sus investigaciones que los docentes carecen, en general, de formación en aspectos relativos a la comunicación.

El objetivo de un buen discurso docente no es sólo mostrar conocimiento y ponerlo a disposición de los alumnos, sino motivar y mover al aprendizaje, lo que hace que el discurso pase de expositivo a persuasivo (Pleyán, 1989). Se pretende convencer (Cros, 2003) de la verdad o validez de las proposiciones, y no sólo hacerlas comprensibles. Muchos han resaltado este carácter argumentativo del discurso del aula (Bruner, 2001; Candela, 2005, 2013; Coll, Onrubia y Mauri, 2008; Cubero, Cubero, Santamaría, et al., 2008; Prados y Cubero Pérez, 2016; Sánchez, García, Rosales, et al., 2008). Además, una estrategia facilitadora del discurso persuasivo docente es la capacidad de poner ejemplos adaptados a los interlocutores. Un trabajo reciente aborda la importancia de trabajar el discurso académico oral y escrito de manera específica en 
el primer curso del Grado de Educación Primaria, para la asignatura de Desarrollo de la Competencia Comunicativa I, a través del aprendizaje basado en Proyectos (Boillos Pereira, 2019).

\section{Factor 2: Adecuación del registro}

La adopción de un registro adecuado supone la elección de la variedad lingüística acorde al contexto y viene dada por cuatro factores: el canal (oral o escrito), el tema, el propósito de la comunicación y el nivel de formalidad (Cassany, Luna y Sanz, 2011). Las comunicaciones propias del ámbito académico se caracterizan por un registro formal, que los futuros docentes deben manejar adecuadamente, aspecto contemplado en diferentes modelos de evaluación de la competencia comunicativa del alumnado del Grado de Maestro o los universitarios en general (Rivera y Vargas, 2015; Tapia, Burdiles y Arancibia, 2003).

En nuestra investigación, se han tenido en cuenta tres indicadores relativos a la adecuación del registro: el reconocimiento del registro formal e informal en la recepción de mensajes, la capacidad de utilizar el registro formal en contextos académicos, y la habilidad para transmitir conocimientos científicos a un público no especializado, adaptando el lenguaje al destinatario. Con respecto a los dos primeros indicadores, se han detectado déficits en el alumnado de Magisterio en cuanto al uso del registro formal, que se plasman en la introducción de construcciones y expresiones propias de un registro coloquial o vulgar en contextos académicos formales (Cicres y Llach, 2014; Níkleva y Núñez, 2013; Rico y Níkleva, 2016; Rivera y Vargas, 2015).

Con respecto al tercer indicador, la capacidad para transmitir conocimientos a diferentes tipos de destinatarios, especializados o no, es una de las competencias aplicables al alumnado de todos los Grados (Luengo, Perea y García, 2013). Además, resulta indispensable para la práctica de la docencia.

\section{Factor 3: Producción eficaz de textos escritos}

Las habilidades de lectura y escritura en contextos académico son también un aspecto esencial en las capacidades comunicativas del alumnado universitario (Martín Villarreal, 2020). En este caso, el factor 3 se centra en la producción de textos escritos en un contexto académico 
formal, en el que el alumnado manifiesta, una vez más, ciertas carencias, sobre todo en adecuación y coherencia del discurso escrito manejo del vocabulario, claridad y precisión y conocimiento de la estructura textual carencias (Corcelles, Cano, Bañales y Vega, 2013; Domingo, Gallego, García y Rodríguez, 2010; Rico y Níkleva, 2016)

En este factor se han incluido tres indicadores: la capacidad de producir diferentes tipos de textos propios del registro académico, el uso eficaz de los párrafos y el empleo de conectores.

El primero supone el conocimiento de la macroestructura y códigos de diferentes géneros textuales del contexto académico universitario (exámenes, trabajos, instancias, correos electrónicos dirigidos a profesores, etc.) y su aplicación en tareas de escritura. Además, la producción de textos académicos requiere un dominio avanzado de la lengua (Cassany, Luna y Sanz 2011) y exige un buen manejo de las habilidades de selección, esquematización y reestructuración de la información, además de suponer un alto grado de especificidad. El alumnado no siempre está familiarizado con los géneros propios del ámbito académico, haciéndose patentes sus dificultades de escritura al afrontar la producción de textos de mayor complejidad, como trabajos fin de máster o grado, artículos o tesis doctorales (Castelló, 2014).

El segundo indicador contribuye a dotar de coherencia al texto, ya que se relaciona con la organización de las ideas. El párrafo estructura el contenido del texto y hace visible su organización (Cassany, Luna y Sanz, 2011), mientras que un mal manejo del párrafo da lugar a escritos desorganizados e inconexos, dificultando la comprensión. También se han señalado deficiencias del alumnado, como el empleo de párrafos demasiado breves o, por el contrario, excesivamente extensos, así como una distribución inadecuada e incoherente de la información (España, 2011).

El tercer indicador incide directamente en la cohesión y coherencia textual que establecen las conexiones lógicas entre las frases, contribuyendo a reforzar la estructura del texto y a organizar las ideas (Errázuriz, 2014). Diversas investigaciones han detectado deficiencias en producciones escritas de los futuros maestros, como la escasez de marcadores textuales, el abuso de la yuxtaposición, la escasa variedad de conectores, o la selección de marcadores inadecuados (Cano-Vela 2016; Errázuriz 2014; España 2011; Rico y Níkleva, 2016). 


\section{Marco empírico}

\section{Objetivos}

De acuerdo con el problema planteado, se definieron los siguientes objetivos:

- Conocer la incidencia del hábito lector de las familias en la percepción de la competencia comunicativa y el nivel inicial de desempeño de los estudiantes del Grado de Maestro en Educación Primaria de las universidades de Jaén y Oviedo.

- Averiguar la incidencia que tiene la titulación de los padres en la percepción de la competencia comunicativa y el nivel inicial de desempeño de los estudiantes del Grado de Maestro en Educación Primaria de las universidades de Jaén y Oviedo.

\section{Contexto y participantes}

Se partió de una población inicial de 726 estudiantes del título de Grado de Educación Primaria de las Universidades de Jaén y Oviedo. La muestra se determinó mediante un muestreo no probabilístico de tipo incidental, en el que el investigador seleccionó a los participantes, en este caso, que quisieron colaborar. La muestra mínima quedo integrada por $(n=252)$ informantes, siendo la muestra suministradora de datos de 361 estudiantes $(49,72 \%)$. Lo que permitió conocer con cierta precisión las características de la población (Otzen y Monterola).

Respondieron 146 hombres (40,5\%) y 215 mujeres (59,5\%), de los cuales 203 procedían de la Universidad de Jaén (56,3\%) y 158 de la de Oviedo (43,7\%). En cuanto al nivel de estudios de los padres, para 109 sujetos (30,19\%) la titulación era Estudios Primarios, para 60 (16,62\%) Bachillerato, para 87 (24,10\%) Formación Profesional, para 39 (10,80\%) Diplomatura, para 51 (14,13\%) Licenciatura o Grado, para 12 (3,32\%) Master; y para $3(0,83 \%)$ Doctorado. Con respecto a los hábitos lectores de las familias, 86 sujetos $(21,6 \%)$ declararon que en su hogar no se lee casi nunca, $148(37,1 \%)$ señalaron que se lee en algunas ocasiones y $146(36,6 \%)$ indicaron que se lee con mucha frecuencia. 


\section{Instrumento de recogida de información}

Se elaboró una escala tipo Likert con una propuesta de 63 ítems de partida, eligiendo este tipo de escala porque cumple con objetividad la función clave de servir de nexo de unión entre los objetivos de la investigación y la realidad de la población estudiada (De Lara, Guijarro y Ballesteros-Velázquez, 2001) y aporta altos niveles de confiabilidad (Vanegas-Rico, Ortega-Andeane, Bustos-Aguayo y Corral-Verdugo, 2018).

La escala se diseñó con cinco opciones de respuesta ("nunca" $=1$, "casi nunca" $=2$, "en algunas ocasiones" $=3$, "casi siempre" $=4$, "siempre" $\left.^{\prime \prime}=5\right)$, siendo las variables de segmentación: "titulación de los padres" y "nivel de afición a la lectura en la familia". En la revisión bibliográfica realizada estas dos variables parecen ser relevantes para generar diferencias significativas en los resultados, por lo que el análisis se centró en ellas y su relación con las tres unidades de competencia mencionadas. La variable dependiente quedó integrada por tres dimensiones: desarrollo de la comunicación oral efectiva en el aula, adecuación del registro lingüístico al contexto y producción eficaz de textos escritos.

\section{Validez y fiabilidad del cuestionario}

La validez de contenido determina en qué medida los ítems de la escala son representativos de las características que están siendo medidas. El cuestionario inicial fue valorado por 9 profesores universitarios, especialistas en el ámbito de las competencias comunicativa y digital. Estos expertos consideraron si el vocabulario era comprensible para la muestra, si los ítems respondían a la finalidad de la investigación y si las preguntas eran cortas y directas. En esta primera fase, el cuestionario inicial de 63 ítems quedó reducido a 54.

Para la validez de constructo se aplicó el análisis de componentes principales que aportó un valor de adecuación muestra $(\mathrm{KMO}=0.882)$ y la esfericidad de Bartlett significativa ( $p=.000)$, tabla 1 ; además, se consideró la correlación entre variables y factores a partir de un peso de 0.50. 
Hábitos lectores y titulación de los padres en la percepción de la competencia comunicativa de futuros maestros

Beatriz Sierra-Arizmendiarrieta y María Del Rosario Neira-Piñeiro

Tabla 1

Factorización de variables.

\begin{tabular}{llr}
\hline Prueba de adecuación muestral, Kaiser-Meyer-Olkin & 0.882 \\
\hline \multirow{3}{*}{ Prueba de Esfericidad de Bartlett } & Aprox. Chi cuadrado & 1894.788 \\
\cline { 2 - 3 } & Gl. & 741 \\
\cline { 2 - 3 } & Sig & 0.000 \\
\hline
\end{tabular}

Nota: $(1){ }^{*} p<.05$.

El análisis factorial de componentes rotados, rotación varimax, definió trece ítems resultantes, distribuidos en 3 factores, con una varianza total explicada del $37,061 \%$ (tabla 2).

Tabla 2

Distribución de las cargas factoriales mediante rotación varimax

\begin{tabular}{lcc}
\hline Factores & $\begin{array}{c}\text { Distribución } \\
\text { de la varianza } \\
(\%)\end{array}$ & Ítems \\
\hline F-1: Desarrollo de la comunicación oral efectiva & 19.499 & $20,21,22$, \\
en el aula & & $23,26,33$ \\
\hline F-2: Adecuación del registro lingüístico al contexto & 9.322 & $7,8,9,15$ \\
\hline F-3: Producción eficaz de textos escritos. 8,240 & 8.240 & $17,28,38$ \\
\hline Varianza total explicada & 37.061 & \\
\hline$p \leq 0.05$. & &
\end{tabular}

La fiabilidad permitió justificar la estabilidad de la escala, siendo el Alpha de Crombach respecto a la totalidad $(\alpha=0.889)$ y las dos mitades, para las pares $(\alpha=0.875)$, mientras que para las impares fue de $(\alpha=0,875)$. Los resultados de los análisis si se elimina cada uno de los ítems, indicaron que reflejan equilibrio [(ítem $7=0.775<0,889=$ total), (ítem 13 $=0.867<0.889=$ total)], por lo que no se eliminó ninguno de los ítems aportados en la validez de constructo.

\section{Método}

Atendiendo a los datos aportados por el problema de investigación y los dos objetivos definidos en la presente investigación, se llevó a cabo un estudio descriptivo-transversal, mediante análisis de datos bivariados, concretamente un análisis contingencia (Inzunza, 2016). En este caso la pregunta de investigación se orienta a conocer cómo inciden dos va- 
riables descriptivas sobre la dependiente y determinar en qué medida están asociadas (Cañadas, Batanero, Contreras y Arteaga, 2011); además, las variables a comparar son ordinales, lo que llevar a cabo el estudio con tablas de contingencia, partiendo de la prueba Chi-cuadrado $\left(\chi^{2}\right)$. Se aceptaron los análisis en los que se rechaza la hipótesis de no asociación o independencia entre variables al nivel de $(p \leq \alpha=0.05)$.

\section{Resultados}

Atendiendo a los datos aportados por los informantes, se realizó el análisis de contingencia $\left[\left(\chi^{2}=19.478\right),(p=0.013 \leq 0.05)\right]$, se rechazándose la hipótesis de no asociación (Tabla 3). Los resultados indican que "comunicación oral efectiva en el aula" es algo asumido por los estudiantes de primero de los estudios del Grado de Maestro, aunque en diferentes porcentajes. "Siempre" la ponen en práctica un 7,9\% de los estudiantes cuyas familias "no leen casi nunca", porcentaje que aumenta en la medida en que las familias de los estudiantes leen con más frecuencia. Concretamente, el $43,1 \%$ de los estudiantes cuyas familias leen en algunas ocasiones" utiliza "casi siempre" la "comunicación oral efectiva", porcentaje que asciende a un $55,4 \%$ en los estudiantes en cuyas familias "se lee con mucha frecuencia". Por consiguiente, la frecuencia con la que se lee en la familia incide notablemente en las habilidades de comunicación oral efectiva en el aula.

Tabla 3

Relación entre la variable hábitos hacia la lectura en la familia y el desarrollo de la comunicación oral efectiva en el aula

\begin{tabular}{|c|c|c|c|c|c|c|c|c|}
\hline & & sarrollo & $\begin{array}{l}\text { de la } \\
\text { fectiva }\end{array}$ & $\begin{array}{l}\text { comun } \\
\text { en el a }\end{array}$ & $\begin{array}{l}\text { icación } \\
\text { ula }\end{array}$ & oral & $\chi^{2}$ & $p$ \\
\hline & & Porce & entajes & & & & & \\
\hline & 1 & 2 & 3 & 4 & 5 & Total & 19.478 & $0.013<0.05$ \\
\hline Hábitos $\frac{1}{1}$ & 0.0 & 16.7 & 27.7 & 15.6 & 7.9 & 16.5 & & \\
\hline lectores $\frac{2}{2}$ & 0.0 & 66,7 & 37.0 & 43.1 & 35.7 & 41.0 & & \\
\hline familia & 100.0 & 16,7 & 38.9 & 40.1 & 55.4 & 42.5 & & \\
\hline Total & 100.0 & 100,0 & 100.0 & 100.0 & 100.0 & 100.0 & & \\
\hline
\end{tabular}

Hábitos lectores: 1 "No se lee casi nunca", 2 "Se lee en algunas ocasiones", 3 "Se lee con mucha frecuencia"/ Nivel de ejercicio de la comunicación oral efectiva en el aula: 1 "Nunca", 2 "Casi nunca, 3 "Con alguna frecuencia", 4"Casi siempre", 5 "Siempre". 
La frecuencia con la que se lee en la familia también incide en la capacidad de los estudiantes para "adecuar el registro lingüístico al contexto" (Tabla 4), [ $\left.\left(\chi^{2}=14.449\right),(p=0.026 \leq 0.05)\right]$, rechazándose la hipótesis de no asociación. El 40,2\% del alumnado procedente de familias que leen en "algunas ocasiones" utilizan el registro lingüístico adecuado al contexto "casi siempre", mientras que el 52,2\% de los sujetos con idéntica situación familiar adoptan el registro adecuado "con alguna frecuencia". Los índices aumentan considerablemente cuando en las familias se "lee con mucha frecuencia", observándose que el 62,5\% de los que están en esta situación "siempre" adecuan el "registro lingüístico al contexto. Sin embargo, la variable explicativa no incide en la adecuación del registro lingüístico al contexto cuando los estudiantes pertenecen a familias en las que no se lee.

Tabla 4

Relación entre la variable hábitos hacia la lectura en la familia y adecuación del registro lingüístico al contexto

\begin{tabular}{|c|c|c|c|c|c|c|c|c|}
\hline & \multicolumn{6}{|c|}{$\begin{array}{c}\text { Adecuación del registro lingüístico al } \\
\text { contexto }\end{array}$} & \multirow[t]{2}{*}{$\chi^{2}$} & \multirow[t]{2}{*}{$p$} \\
\hline & & Porce & entajes & & & & & \\
\hline \multirow{5}{*}{$\begin{array}{l}\text { Hábitos } \\
\text { lectores } \\
\text { en la } \\
\text { familia }\end{array}$} & 1 & 2 & 3 & 4 & 5 & Total & 14.449 & $0.026<.05$ \\
\hline & 15.4 & 14.5 & 15.9 & 18.4 & 17.5 & 16.5 & & \\
\hline & 23.1 & 38.7 & 52.2 & 40.2 & 20.0 & 41.0 & & \\
\hline & 61.5 & 46.8 & 31.9 & 41.4 & 62.5 & 42.5 & & \\
\hline & 100.0 & 100.0 & 100.0 & 100.0 & 100.0 & 100.0 & & \\
\hline
\end{tabular}

Hábitos lectores: 1 "No se lee casi nunca", 2 "Se lee en algunas ocasiones", 3 "Se lee con mucha frecuencia"/Adecuación del registro lingüístico al contexto: 1 "Nunca", 2 "Casi nunca", 3"Con alguna frecuencia, 4"Casi siempre", 5 "Siempre".

Respecto a la "producción eficaz de textos escritos" (Tabla 5), $\left[\left(\chi^{2}=24.003\right),(p=0.022 \leq 0.05)\right]$, tan solo discrimina la variable explicativa en las ocasiones en las que en las familias de los estudiantes "se lee con mucha frecuencia". Un 56,6\% de los estudiantes que afirman que sus familias "leen con mucha frecuencia", utiliza "siempre" la "producción eficaz de textos escritos". Se puede afirmar, pues, que en las familias lectoras se desarrolla más la competencia comunicativa entre los hijos, referida en este caso a la capacidad de producción eficaz de textos escritos. 
Tabla 5

Relación entre la variable hábitos hacia la lectura en la familia y producción eficaz de textos escritos

\begin{tabular}{|c|c|c|c|c|c|c|c|c|c|}
\hline \multicolumn{8}{|c|}{ Producción eficaz de textos escritos } & \multirow{2}{*}{$\chi^{2}$} & \multirow{2}{*}{$p$} \\
\hline & & & Porce & entajes & & & & & \\
\hline \multirow{5}{*}{$\begin{array}{l}\text { Hábitos } \\
\text { lectores } \\
\text { en la } \\
\text { familia }\end{array}$} & & 1 & 2 & 3 & 4 & 5 & Total & 24,003 & $0,002<.05$ \\
\hline & 1 & 0.0 & 20.0 & 20.0 & 18.9 & 12.1 & 16.5 & & \\
\hline & 2 & 100.0 & 50.0 & 57.8 & 44.1 & 29.3 & 41.0 & & \\
\hline & 3 & 0.0 & 30.0 & 22.2 & 37.1 & 56.6 & 42.5 & & \\
\hline & Total & 100.0 & 100.0 & 100.0 & 100.0 & 100.0 & 100.0 & & \\
\hline
\end{tabular}

Hábitos lectores: 1 "No se lee casi nunca", 2 "Se lee en algunas ocasiones", 3"Se lee con mucha frecuencia"/Adecuación del registro lingüístico al contexto: 1 "Nunca", 2 "Casi nunca", 3"Con alguna frecuencia", 4 "Casi siempre", 5"Siempre".

En cambio, no incide de modo efectivo la variable explicativa "nivel de estudios de los padres" (Tabla 6), de hecho se rechaza la hipótesis de no asociación $\left[\left(\chi^{2}=20.057\right),(p=0.695 \leq 0.05)\right]$, ya que los estudiantes cuyos padres o madres tienen el título de "Formación Profesional" son los que más utilizan "el desarrollo de la comunicación oral efectiva en el aula". Un 25\% de los sujetos con esta situación familiar lo hacen "siempre", seguidos por aquellos estudiantes cuyos padres son "licenciados o graduados" $(23,2 \%)$, estando los hijos de las familias que poseen "master o doctorado" en los últimos lugares. Los datos aportados por $\chi^{2}=20,057$ y el valor de $p=0,695>0,05$ evidencian que no hay incidencia significativa del mayor nivel de titulación de los padres en la unidad de competencia objeto de análisis. 
Hábitos lectores y titulación de los padres en la percepción de la competencia comunicativa de futuros maestros

Beatriz Sierra-Arizmendiarrieta y María Del Rosario Neira-Piñeiro

Tabla 6

Relación entre la variable titulación más alta de los padres y el desarrollo de la comunicación oral efectiva en el aula

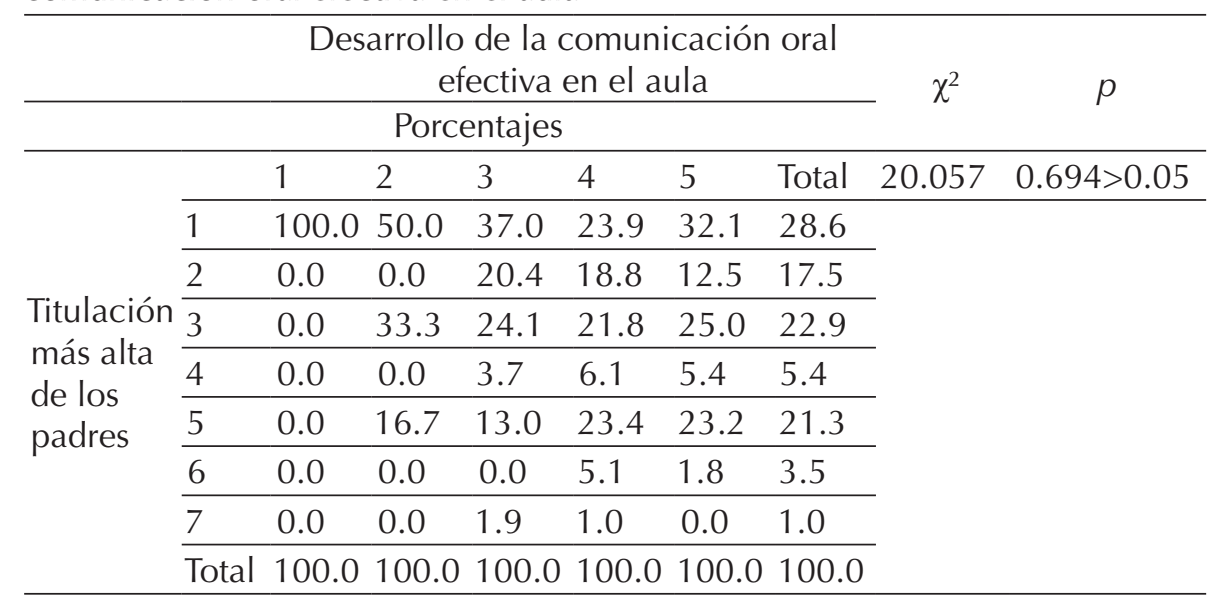

Titulación más alta de los padres: 1 Estudios Primarios, 2 Bachiller, 3 Formación Profesional, 4 Diplomatura, 5 Licenciatura o Grado, 6 Master, 7 Doctorado/ Desarrollo de la comunicación efectiva en el aula: 1 "Nunca", 2 "Casi nunca", 3 "Con alguna frecuencia", 4 "Casi siempre", 5 "Siempre".

De igual modo, el análisis de contingencia efectuado entre la variable explicativa y el hecho de que los estudiantes utilicen "el registro lingüístico adecuado al contexto" pone de manifiesto que no hay incidencia entre el uso de la unidad de competencia referenciada y la mayor titulación académica de los padres de los estudiantes [ $\left.{ }^{2}=18.685\right)$, $(p=0.769>0.05)]$ aceptando la hipótesis de no asociación, (Tabla 7). Así, los mayores porcentajes en la percepción de esta unidad de competencia aparecen en el alumnado cuyos padres tienen "Estudios primarios", que manifiestan utilizar "casi siempre" (30,1\%) o "siempre" $(28,4 \%)$ el registro adecuado al contexto. Quedan en segundo lugar los estudiantes cuyos padres han cursado "Formación Profesional", quienes dicen utilizar el registro lingüístico adecuado al contexto "casi siempre" (24,5\%) o "siempre" (24,1\%). Ello evidencia que el nivel académico de los padres no incide en la frecuencia de ejercicio de esta unidad de competencia. 
Tabla 7

Relación entre la variable titulación más alta de los padres y adecuación del registro lingüístico al contexto

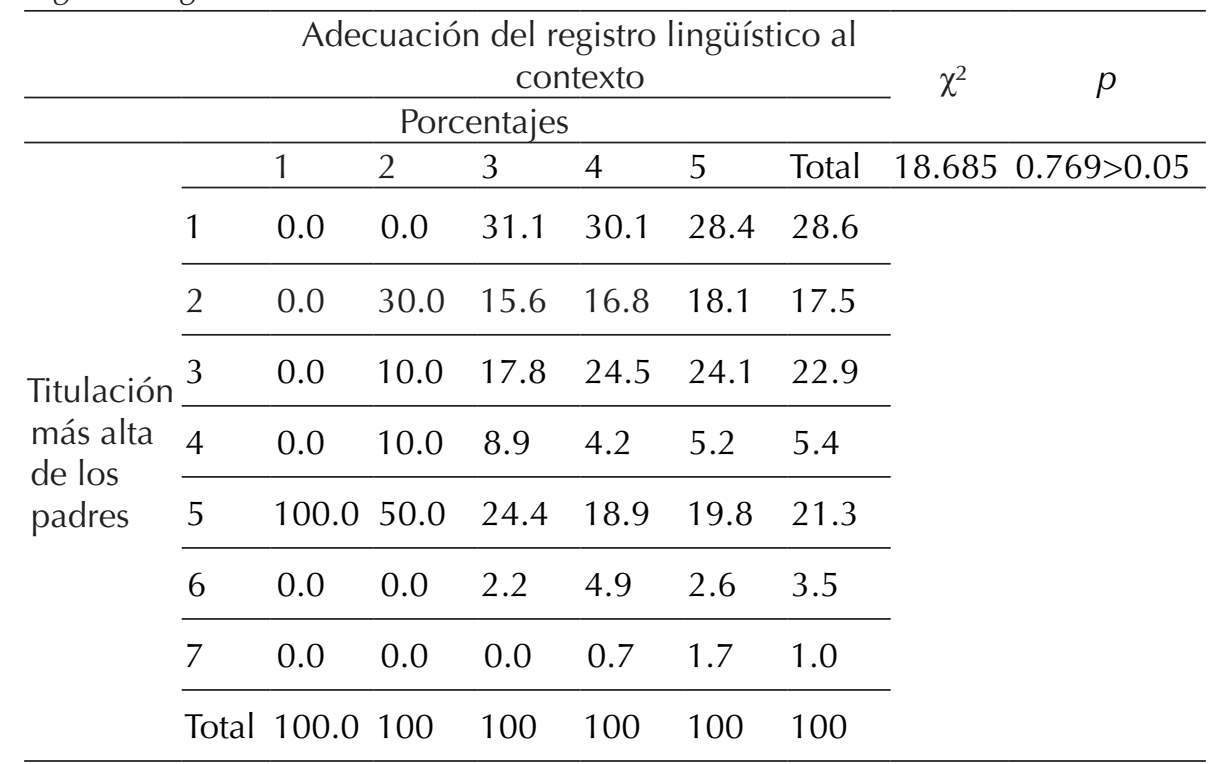

1 Estudios primarios, 2 Bachiller, 3 Formación Profesional, 4 Diplomatura, 5 Licenciatura o Grado, 6, Master, 7 Doctorado/Adecuación del registro lingüístico al contexto: 1 "Nunca", 2 "Casi nunca", 3 "Con alguna frecuencia", 4 "Casi siempre", 5 "Siempre".

Tampoco la "titulación más alta de los padres" mantiene una relación positiva con la "producción eficaz de textos escritos, en los estudiantes del Grado de Maestro (Tabla 8), ya que no hay relación entre titulaciones más altas y la "producción eficaz de textos escritos", $\left[\left(\chi^{2}=58.527\right),(p=0.676 \leq 0.05)\right]$ aceptando la hipótesis de no asociación. En este caso, son los estudiantes cuyos padres tienen "Estudios primarios" y de "Formación Profesional" los que afirman en porcentajes más elevados $(40,0 \%$ y $27,5 \%$, respectivamente), mostrar "siempre" una "producción eficaz de textos escritos". 
Hábitos lectores y titulación de los padres en la percepción de la competencia comunicativa de futuros maestros

Beatriz Sierra-Arizmendiarrieta y María Del Rosario Neira-Piñeiro

Tabla 8

Relación entre la variable titulación más alta de los padres y producción eficaz de textos escritos

\begin{tabular}{|c|c|c|c|c|c|c|c|c|c|}
\hline \multicolumn{8}{|c|}{ Producción eficaz de textos escritos } & \multirow{2}{*}{$\chi^{2}$} & \multirow{2}{*}{$p$} \\
\hline \multirow{2}{*}{\multicolumn{10}{|c|}{$\frac{\text { Porcentajes }}{2}$}} \\
\hline & & & & & & & & & \\
\hline \multirow{8}{*}{$\begin{array}{l}\text { Titulación } \\
\text { más alta } \\
\text { de los } \\
\text { padres }\end{array}$} & 1 & 7.7 & 17.7 & 23.0 & 41.4 & 40.0 & 28.6 & & \\
\hline & 2 & 30.8 & 16.1 & 25.7 & 11.5 & 5.0 & 17.5 & & \\
\hline & 3 & 23.1 & 24.2 & 18.6 & 25.3 & 27.5 & 22.9 & & \\
\hline & 4 & 15.4 & 11.3 & 1.8 & 6.9 & 0.0 & 5.5 & & \\
\hline & 5 & 23.1 & 22.6 & 28.3 & 13.8 & 15.0 & 21.3 & & \\
\hline & 6 & 0.0 & 8.1 & 0.9 & 1.1 & 10.0 & 3.5 & & \\
\hline & 7 & 0,0 & 0,0 & 1,8 & 0,0 & 2,5 & 1,0 & & \\
\hline & Total & 100,0 & 100,0 & 100,0 & 100,0 & 100,0 & 1,00 & & \\
\hline
\end{tabular}

Titulación más alta de los padres: 1 Estudios Primarios, 2 Bachiller, 3 Formación Profesional, 4 Diplomatura, 5 Licenciatura o Grado, 6 Master, 7 Doctorado/ Producción eficaz de textos escritos: 1 "Nunca", 2 "Casi nunca", 3 "Con alguna frecuencia", 4 "Casi siempre", 5 "Siempre".

\section{Discusión y conclusiones}

A lo largo de estas páginas, se ha aludido a una variada selección de bibliografía, por lo que el interés de la competencia comunicativa no deja lugar a dudas. Muchas investigaciones sitúan a la lectura y al hábito lector como una de las variables que en mayor medida favorecen los resultados académicos, encontrándose propuestas recientes para potenciar dicho hábito en la universidad (Elche, Sánchez-García y Yubero, 2019; Sánchez-García, 2019). En la presente investigación se ha querido analizar la incidencia del hábito lector en la familia y la titulación de los padres en la percepción de tres unidades de la competencia comunicativa (comunicación oral efectiva en el aula, adecuación del registro y producción eficaz de textos escritos) de estudiantes de primer curso del Grado de Maestro en Educación Primaria. Para ello, se pasó un cuestionario ad hoc de 54 ítems a 361 estudiantes del citado curso de las Universidades de Jaén y Oviedo.

Los resultados evidencian que la frecuencia con la que se lee en la familia incide en el nivel de competencia comunicativa de los futuros maestros en las universidades seleccionadas. La influencia de un entor- 
no familiar lector en la configuración de los hábitos y actitudes hacia la lectura en los menores ha sido probada por diferentes estudios (Yubero y Larrañaga, 2010; Serna, Rodríguez y Etxániz, 2017), así como su repercusión en la competencia lectora y, de modo más general, en el rendimiento escolar (Gil, 2009). En esta investigación, se comprueba el impacto positivo del hábito lector de la familia en las tres unidades de competencia definidas (comunicación oral, adecuación del registro al contexto y producción de textos escritos). A la vista de los resultados, cabe destacar la importancia de las prácticas lectoras en el hogar, cuyo influjo va más allá de la infancia y adolescencia, extendiéndose a la edad adulta. En este sentido, algunos trabajos sugieren la relevancia de las experiencias familiares en torno a la lectura vividas en la infancia, y su incidencia posterior en la conformación del perfil lector de los futuros docentes (Dueñas, Tabernero, Calvo y Consejo, 2014).

Por el contrario, los resultados no muestran que haya relación entre el nivel de estudios de los padres y las unidades de la competencia comunicativa analizadas. En este sentido, no hay coincidencia con investigaciones realizadas con menores, donde se manifiesta la incidencia de esta variable en el rendimiento académico de los estudiantes (Bravo et al., 2017; Córdoba et al., 2011), o en el hábito lector. Así, el bajo nivel académico de los padres puede dificultar los hábitos lectores en los niños (Moreno-Sánchez, 2001). También en una reciente investigación sobre el rendimiento académico de los estudiantes de educación secundaria se encuentra una relación clara entre el rendimiento académico y el nivel de educación de los padres, demostrándose que el nivel educativo de la madre es el que tiene mayor incidencia en el rendimiento académico del estudiante (Rodríguez Rodríguez y Guzmán Rosquete, 2019). Asimismo, respecto al rendimiento académico de estudiantes universitarios, otra investigación muestra que el nivel educativo de la madre, cuando es superior a licenciatura, influye positivamente en dicho rendimiento, no resultando significativo estadísticamente el nivel educativo del padre (Espejel García y Jiménez García, 2019). La discrepancia entre las investigaciones mencionadas y nuestros resultados puede quizá deberse a las variables que se hayan tenido en cuenta para valorar el rendimiento académico. En nuestro caso se han centrado exclusivamente en los aspectos mencionados de la competencia comunicativa, lo que podría explicar la diferencia en los resultados.

Nuestros datos concuerdan más con una investigación sobre rendi- 
miento académico en estudiantes de los programas de Psicología de Instituciones de Educación Superior de la ciudad de San Juan de Pasto (Guevara, Jaramillo y Tovar, 2013). En ella concluyen que no existe correlación significativa entre la variable "rendimiento académico" y el nivel educativo de los padres. Ello se puede explicar por el mayor nivel de independencia de los hijos en esa etapa, lo que hace que la influencia y el seguimiento constante de los padres en sus tareas disminuya y su mayor o menor preparación no se aprecie tanto.

Creemos que es de vital importancia la diferencia entre el rendimiento académico, para lo que efectivamente la influencia del padre o madre puede ser relevante, y el nivel de desarrollo de las competencias. Éstas precisan de práctica constante y, por ello, de acuerdo con los resultados obtenidos en esta investigación, "el hábito lector de la familia" puede ayudar a adquirir el mismo hábito en los estudiantes desde su infancia, lo que tiene una gran conexión con la práctica oral, la comunicación escrita y la adecuación del registro. Se puede suponer que la práctica continuada ha ido progresivamente mejorando dichas unidades de competencia. Por el contrario, el hecho de que la variable "titulación de los padres" no tenga la misma incidencia puede deberse precisamente a que no es una variable que sirva de modelo en el ejercicio, sino, por decirlo, así una variable 'estática' respecto al desarrollo competencial. Esto es, puede servir como ayuda en las tareas de los estudiantes e incluso como elemento motivador para seguir estudiando, pero no está directamente relacionada con la práctica de la competencia analizada, que depende exclusivamente del estudiante en la etapa universitaria.

Respecto a futuras líneas de investigación, este estudio se ha centrado en tres indicadores de la competencia comunicativa relacionados con la producción de textos -tantos orales como escritos- que resultan de especial relevancia para el ejercicio de la docencia. La investigación podría ampliarse en el futuro con el estudio de las habilidades lectoras de los futuros docentes y su perfil lector, en relación con las mismas variables comentadas. 
Hábitos lectores y titulación de los padres en la percepción de la competencia comunicativa de futuros maestros

Beatriz Sierra-Arizmendiarrieta y María Del Rosario Neira-Piñeiro

\section{Referencias}

Aguilar, A. (2013). Competencia comunicativa y norma en los grados de maestro en Educación Primaria. Estudios sobre el Mensaje Periodístico, 19, 25-33.

Alarcón Montiel, E.; Piña Osorio, J.M; García Valcárcel, A. y Tejedor, F.J. (2019). Perfiles de socialización familiar en estudiantes universitarios de alto rendimiento académico. Perfiles educativos, 41(165), 62-80. https://dx.doi.org/10.22201/ iisue.24486167e.2019.165.58742

Boillos Pereira, M.M. (2019). Alfabetización académica temprana a través del aprendizaje basado en proyectos. Lenguaje y Textos, [S.I.], 50, 143-154, ISSN 2530-0075. Disponible en: <https://polipapers.upv.es/index.php/lyt/article/view/11421>. doi:https:// doi.org/10.4995/lyt.2019.11421.

Bravo, M.; Salvo, S.; Mieres, M.; Mansilla, J. y Hederich, J. (2017). Perfiles de desempeño académico: la importancia de las expectativas familiares. Perfiles Latinoamericanos, 25(50), 361-386. Doi: 10.18504/pl2550-016-2017

Bruner, J.S. (2001). Self-making and World-making. En J. Brockmeier y D. Carbaugh (eds.), Narrative and identity: Studies in Autobiography, Self and Culture (pp. 25-37). Amsterdam: John Benjamins.

Camacho, O. y Sáenz, O. (2000). Técnicas de comunicación eficaz para profesores y formadores. Alcoy: Marfil.

Candela, A. (2005). Students Participation as Co-authoring of School Institucional Practices. Culture and Psychology, 11(3), 321-337.

Candela, A. (2013). La construcción discursiva del conocimiento científico en el aula. Educación y Educadores, 16(1), 41-65.

Cano-Vela, A. G. (2016). El uso de marcadores del discurso en textos escritos por estudiantes del grado de Maestro y de la especialidad de lengua castellana y literatura del MUFPS. En A. Díez, V. Brotons, D. Escandell y J. Rovira (Eds.), Aprendizajes plurilingües y literarios. Nuevos enfoques didácticos (pp. 419-427). Alicante: Publicaciones de la Universidad de Alicante.

Cañadas, G., Batanero, C, José Miguel Contreras, J. M. y Pedro Arteaga, P. (2011). Estrategias en el estudio de la asociación de tablas de contingencia por estudiantes de psicología. Educación Matemática, 23(2), 1 - 31. Recuperado de http://www.scielo. org.mx/pdf/ ed/ v23n2/v23n2a2.pdf

Cassany, D. Luna, M. y Sanz, G. (2011). Enseñar lengua. Barcelona: Graó.

Castellà. J. M., Comelles, S., Cros, A. y Vilà, M. (2007). Entender(se) en clase. Barcelona: Graó.

Castelló, M. (2014). Los retos actuales de la alfabetización académica: estado de la cuestión y últimas investigaciones. Enunciación, 19(2), 346-365.

Castro, M.; Expósito-Casas, E.; López-Martín, E.; Lizasoain, L.; Navarro-Asencio, E. y Gaviria, J. L. (2015). Parental involvement on student academic achievement: A metaanalysis. Educational Research Review, 14, 33-46. doi: 0.1016/j.edurev.2015.01.002

Cicres, J. y Llach, S. (2014). Análisis de las creencias hacia el registro formal y la variedad estándar en la formación inicial de los maestros, Tonos Digital, 26, 1-22.

Clemente-Ricolfe, J.S. y Escribá-Pérez, C. (2013). Análisis de la percepción de las compe- 
Hábitos lectores y titulación de los padres en la percepción de la competencia comunicativa de futuros maestros

Beatriz Sierra-Arizmendiarrieta y María Del Rosario Neira-Piñeiro

tencias genéricas adquiridas en la universidad. Revista de Educación, 362, 535-561. Doi: 10.4438/1988-592X-RE-2013-362-241.

Coll, C., Onrubia, J. y Mauri, T. (2008). Ayudar a aprender en contextos educativos: el ejercicio de la influencia educativa y el análisis de la enseñanza. Revista de educación, 346, 33-70.

Conchado, A. y Carot, J.M. (2013). Puntos fuertes y débiles en la formación por competencias según los graduados universitarios españoles, Revista de Docencia Universitaria, 11(1), 429 - 446.

Corcelles, M. Cano, M., Bañales, G. y Vega, N. (2013). Enseñar a escribir textos científico-académicos mediante la revisión colaborativa: El trabajo final de grado en Psicología. REDU, 11(1), 79-104.

Córdoba, L. G.; García, V.; Luengo, LM; Vizuete, M. y Feu, S. (2011). Determinantes socioculturales: su relación con el rendimiento académico en alumnos de Enseñanza Secundaria Obligatoria. Revista de Investigación Educativa, 29 (1), 83-96.

Cros, A. (2003). Convencer en clase. Argumentación y discurso docente. Barcelona: Ariel.

Cuadrado, I. (2008). ¿Son conscientes los profesores de secundaria de los recursos comunicativos verbales y no-verbales que emplean en el aula? Revista Iberoamericana de Educación, 46(6), 1-13.

Cubero, R., Cubero, M., Santamaría, A., De la Mata, M., Ignacio, M.J., y Prados, M. M. (2008). La educación a través de su discurso. Revista de Educación, 346, 71-104.

De Lara, E. y Ballesteros-Velázquez, B (2001). Métodos de investigación en educación social. Madrid: UNED.

Domingo, J., Gallego, J.L., García, I. y Rodríguez, A. (2010). Competencias comunicativas de maestros en formación. Profesorado. Revista de curriculum y formación del profesorado, 14(2) 303-323.

Domínguez, M.C.; Medina, A. y López, E. (2018). Desarrollo de competencias en el primer curso de universidad: estudio de caso, Publicaciones, 48(1), 39-61. doi: http:// dx.doi.org/10.30827/publicaciones.v48i1.7325.

Dueñas, J. D., Tabernero, R.; Calvo V. y Consejo, E. (2014). La lectura literaria ante nuevos retos: canon y mediación en la trayectoria lectora de futuros profesores. Ocnos, 11, 21-43. doi: http://dx.doi.org/10.18239/ocnos_2014.11.02

Elche, M.; Sánchez-García, S. y Yubero, S. (2019). Lectura, ocio y rendimiento académico en estudiantes universitarios del área socioeducativa. Educación XX1, 22(1), 215237. https://doi.org/10.5944/educxx1.21548

Errázuriz, M. C. (2014). El desarrollo de la escritura argumentativa académica: los marcadores discursivos. Onomázein, 30, 217-236. doi: 10.7764/onomazein.30.13

España, E. (2011). Enseñanza de la escritura en el ámbito universitario: situación actual y perspectivas. Normas. Revista de Estudios Lingüísticos Hispánicos, 1, 37-51.

Espejel García, M. V., y Jiménez García, M. (2019). Nivel educativo y ocupación de los padres: Su influencia en el rendimiento académico de estudiantes universitarios. RIDE Revista Iberoamericana Para La Investigación Y El Desarrollo Educativo, 10(19). https://doi.org/10.23913/ride.v10i19.540

Fajardo, F.; Maestre, M.; Felipe, E.; León, B. y Polo M.I. (2017). Análisis del rendimiento 
Hábitos lectores y titulación de los padres en la percepción de la competencia comunicativa de futuros maestros

Beatriz Sierra-Arizmendiarrieta y María Del Rosario Neira-Piñeiro

académico de los alumnos de Educación Secundaria Obligatoria según las variables familiares. Educación XX1, 20(1), 209-232. Doi: 10.5944/educXX1.14475

Gallego, J.L. y Rodríguez, A. (2014). Percepción del alumnado universitario de Educación Física sobre su competencia comunicativa. Movimento, 20(2), 425-444.

Gallego, J.L. y Rodríguez, A. (2015). Competencias comunicativas de maestros en formación de educación especial. Educación y educadores, 18(2), 209-225. Doi: 10.5294/ edu.2015.18.2.2

García-Fontes, W. (2012). Efectos de los hábitos de lectura familiares sobre los resultados académicos en PIRLS 2011. En Ministerio de Educación, Cultura y Deporte (Ed.), PIRLS-TIMSS 2011. Estudio internacional de progreso en comprensión lectora, matemáticas y ciencias. Volumen II: Madrid: IEA.

Gil, J. (2009). Hábitos y actitudes de las familias hacia la lectura y competencias básicas del alumnado. Revista de Educación, 350, 301-322.

Guevara, E.P.; Jaramillo, R. y Tovar, S. (2013). Factores familiares y su relación con el rendimiento académico en estudiantes de psicología, Revista Virtual Universidad Católica del Norte, 40, 122-140.

Gumperz, J. y Hymes, D. (Eds.) (1972). Directions on Sociolinguistics. The Ethnography of Communication. New York: Holt, Rinehart and Winston.

Guzmán-Simón, F. y García-Jiménez, E. (2014). Los hábitos lectoescritores en los alumnos universitarios. Revista Electrónica Interuniversitaria de Formación del Profesorado, 17(3), 79-92. doi: 10.6018/reifop.17.3.204071

Hymes, D. (1971). On Communicative Competence. Philadelphia: University of Pensylvania Press.

Inzunza, S. (2016) Análisis de datos bivariados en un ambiente basado en applets y software dinámico. Educación Matemática, 28(3), 61-89. http://dx.doi.org/10.24844/ em3003.07

Luengo, Perea y García. (2013). Producción textual en la universidad: una investigación para la innovación docente. Lenguaje y Textos, 38, 125-132.

Margallo, A.M. y Mata, J. (2015). La lectura: práctica social y formación escolar. Lectura, sociedad y escuela. En J. Mata, P. Núñez y J. Rienda (Coords. y Eds.), Didáctica de la lengua y la literatura (pp.179-202). Madrid: Pirámide.

Martín Villarreal, J. P. (2020). Iniciativas de fomento de la lectoescritura en el ámbito universitario. Álabe, 21, 1-15. doi: 10.15645/Alabe2020.21.4

Mayoral, S., Timoneda, C. y Pérez, F. (2013). Evaluación de los procesos metacognitivos en estudiantes de Grado en maestro de Educación Infantil y Primaria en tareas de lectura. Aula Abierta, 41 (3), 5-12.

McCroskey, J., y Richmond, V. (2006). Introduction to Communication in the Classroom: The Role of Communication in Teaching and Training. Boston: Allyn \& Bacon.

Miller, P. (2005). Body Language: An Illustrated Introduction for Teacher. Chicago: Patrick. W. Miller and Associates.

Moreno Sánchez, E. (2001). Análisis de la influencia de la familia en los hábitos lectores de sus hijas e hijos. Un estudio etnográfico. Contextos educativos, 4, 177-196.

Níkleva, D.G. y López-García, M.P. (2019). El reto de la expresión oral en Educación 
Hábitos lectores y titulación de los padres en la percepción de la competencia comunicativa de futuros maestros

Beatriz Sierra-Arizmendiarrieta y María Del Rosario Neira-Piñeiro

Primaria: características, dificultades y vías de mejora. Educatio Siglo XXI, 37(3), 9-32. doi: http://doi.org/10.6018/educatio.399141

Níkleva, D.G. y Núñez M.P. (2013). El correo electrónico como género discursivo: percepciones y habilidades para su composición en estudiantes universitarios. Revista Española de Lingüística Aplicada, 26, 385-407.

Otzen, T.; Monterola, C. (2017). Técnicas de muestreo sobre una población a estudio. I. J. of Morph 35(1) 227-232. Recuperado de https://scielo.conicyt.cl/pdf/ ijmorphol/ v35n1/ art37.pdf

Pleyán, C. (1989). El discurso docente, una actividad persuasiva. Cuadernos de Pedagogía, 175, 16-19.

Prados, M.M. y Cubero, M. (2016). ¿Cómo argumentan docentes y discentes en las aulas universitarias? Educación XX1, 19(1), 115-134. doi:10.5944/educXX1.13939.

Rico y Níkleva, D.G. (2016). Análisis de la competencia lingüístico-discursiva escrita de los alumnos de nuevo ingreso del Grado de Maestro en Educación Primaria. Signos, 49(90), 48-70. Doi: 10.4067/S0718-09342016000100003

Rivera, P. y Vargas, J. M. (2015). Las pruebas de diagnóstico como herramienta para analizar la competencia comunicativa escrita de los alumnos de nuevo ingreso de los grados en educación infantil y primaria. En X. Núñez et al. (Eds.), Horizontes Científicos y Planificación Académica en la Didáctica de Lenguas y Literaturas (pp. 1069-1088). Braga: Universidade do Minho.

Rodríguez, A.; Ayllón, M.F.; Gallego, J. L. y Gómez, I.A. (2017). The Communication Skills of Future Teachers during their Initial Training. Multidisciplinary Journal of Educational Research, 7(1), 88-118. doi:10.17583/remie.2017.2200

Rodríguez, M. R. (2011). Diagnóstico y mejora de la competencia comunicativa en los alumnos del grado de primaria. En I Jornadas de Innovación Docente. Sevilla: Universidad de Sevilla.

Rodríguez Rodríguez, D. y Guzmán Rosquete, R. (2019). Rendimiento académico y factores sociofamiliares de riesgo. Variables personales que moderan su influencia. Perfiles educativos, 41(164), 118-134. https://dx.doi.org/10.22201/ iisue.24486167e.2019.164.58925

Romero, M.R.; Castejón-Oliva, F.J.; López, V.M. y Fraile, A. (2017). Evaluación formativa, competencias comunicativas y TIC en la formación del profesorado. Comunicar, 52(XXV), 73-82. doi: https://doi.org/10.3916/C52-2017-07.

Sánchez, E., García, J.R., Rosales, J., et al. (2008). Elementos para analizar la interacción entre estudiantes y profesores: ¿qué ocurre cuando se consideran diferentes dimensiones y diferentes unidades de análisis? Revista de Educación, 346, 105-136.

Sánchez-García, S. (2019). Lectura y rendimiento académico. Actuaciones desde la biblioteca universitaria". Anuario ThinkEPI, 13, e13b03. https://doi.org/10.3145/thinkepi.2019.e13b03

Sanz, G. (2005). Comunicación efectiva en el aula. Barcelona: Graó.

Serna, M., Rodríguez, A., \& Etxaniz, X. (2017). Biblioteca escolar y hábitos lectores en los escolares de Educación Primaria. Ocnos, 16(1), 18-49. doi: 10.18239/ocnos_2017.16.1.1205

Tapia, M., Burdiles, G. y Arancibia, B. (2003). Aplicación de una pauta diseñada para 
Hábitos lectores y titulación de los padres en la percepción de la competencia comunicativa de futuros maestros

Beatriz Sierra-Arizmendiarrieta y María Del Rosario Neira-Piñeiro

evaluar informes académicos universitarios, Signos, 36(54), 249-257. doi: 10.4067/ S0718-09342003005400009

Vanegas-Rico, M. C., Ortega-Andeane, P., Bustos-Aguayo, J. M. y Corral-Verdugo, V. (2018). Desarrollo de la Escala Expectativa de Comportamiento Ambiental de Otros con adultos jóvenes mexicanos. Universitas Psychologica, 17(2), 1-10. doi: https:// doi.org/10.11144/Javeriana.upsy17-2.deec

Vilà, M. y Castellà, J.M. (2014). Enseñar la competencia oral en clase. Aprender a hablar en público. Barcelona: Graó.

Yubero, S. y Larrañaga, E. (2010). El valor de la lectura en relación con el comportamiento lector. Ocnos, 6, 7-20. doi: http://dx.doi.org/10.18239/ocnos_2010.06.01 
